\title{
Extrapolating continuous color emotions through deep learning
}

\author{
Vishaal Ram $\odot,{ }^{1}$ Laura P. Schaposnik $\odot,{ }^{2, *}$ Nikos Konstantinou $\odot,{ }^{3}$ Eliz Volkan $\odot,{ }^{4}$ Marietta Papadatou-Pastou $\odot,{ }^{5}$ \\ Banu Manav, ${ }^{6}$ Domicele Jonauskaite, ${ }^{7}$ and Christine Mohr ${ }^{7}$ \\ ${ }^{1}$ Milton High School, Milton, Georgia 30004, USA \\ ${ }^{2}$ Department of Mathematics, Statistics and Computer Science, University of Illinois, Chicago, Illinois 60607, USA \\ ${ }^{3}$ Department of Rehabilitation Sciences, Faculty of Health Sciences, Cyprus University of Technology, Limassol 3036, Cyprus \\ ${ }^{4}$ Department of Psychology, Cyprus International University, Nicosia 99258, Cyprus \\ ${ }^{5}$ National and Kapodistrian University of Athens, Athens 157 72, Greece \\ ${ }^{6}$ Kadir Has University, Faculty of Art and Design, Department of Interior Architecture and Environmental Design, \\ Kadir Has Caddesi 34083 Cibali-Istanbul \\ ${ }^{7}$ Institute of Psychology, University of Lausanne, Lausanne 1015, Switzerland
}

(Received 6 June 2020; accepted 29 July 2020; published 2 September 2020)

\begin{abstract}
By means of an experimental dataset, we use deep learning to implement an RGB (red, green, and blue) extrapolation of emotions associated to color, and do a mathematical study of the results obtained through this neural network. In particular, we see that males (type- $m$ individuals) typically associate a given emotion with darker colors, while females (type- $f$ individuals) associate it with brighter colors. A similar trend was observed with older people and associations to lighter colors. Moreover, through our classification matrix, we identify which colors have weak associations to emotions and which colors are typically confused with other colors.
\end{abstract}

DOI: 10.1103/PhysRevResearch.2.033350

\section{INTRODUCTION}

The relation between colors and human emotion has been studied for more than a century (see, for instance, [1-8]). Even longer ago, colors were commonly associated to emotions in a universal manner that allowed populations to quickly understand the given emotions. For example, for centuries, in many cultures it has been said that someone "had the blues" [9] or "is feeling blue" when being depressed or sad. As explained in [10], the phrase "feeling blue" comes from deepwater sailing ships: If a ship lost the captain or any of the officers during its voyage, then blue flags would be shown and a blue band would be painted along the entire hull when returning to home port.

Inspired by [11,12], we consider their database [12] to analyze the correlation between colors and emotions via a deep-learning approach. While machine-learning techniques have been used before in this direction (e.g., see [13] and references therein), we take a different approach which allows us to discern several interesting patterns.

When using a deep-learning approach to quantify coloremotion associations, one expects to observe certain behaviors. In particular:

(a) Certain colors should have strong associations with emotion and high classification accuracy.

\footnotetext{
*Corresponding author: schapos@uic.edu

Published by the American Physical Society under the terms of the Creative Commons Attribution 4.0 International license. Further distribution of this work must maintain attribution to the author(s) and the published article's title, journal citation, and DOI.
}

(b) Some colors are associated with multiple emotions and would have a low classification accuracy.

(c) Regional and geographic factors may have a factor in color-emotion associations and would provide a deep-learning approach to distinguish the region.

(d) Several colors are associated with a single emotion.

Color-association studies usually consider a discrete number of colors. In particular, this is the case of the study leading to the dataset [12] which we shall use in the present paper, where participants associated emotions to 12 color terms: red, orange, yellow, green, blue, turquoise, purple, pink, brown, black, gray, and white. It should be emphasized that the experiment did not show colors, but rather gave the terms of colors, and it was left to the participant's imagination as to the choice of what those words meant.

To carry out our mathematical study, we have used the standard Decimal Code (R,G,B) to represent the 12 colors of [12], a depiction of which is in Fig. 1.

In recent decades, colors have also been studied in terms of emotional reactions to color hue, saturation, and brightness (e.g., $[14,15])$. Here, we shall put the two approaches together to consider a different path where we let the color association within our neural network take a continuum of colors, and hence consider a continuous RGB analysis [16], depicted in Fig. 2.

After introducing some background in Sec. II, we dedicate Secs. III and IV to the main findings of our work, which can
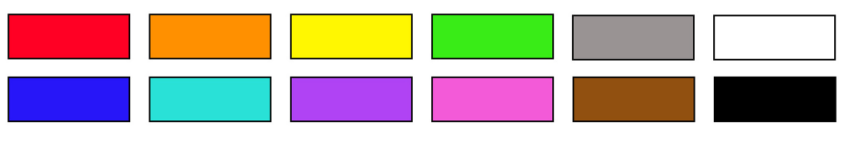

FIG. 1. A depiction of the 12 colors used in [12]. 


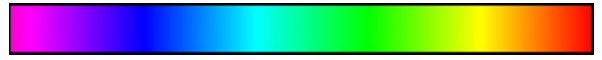

FIG. 2. A depiction of the continuous RGB palate used in the present paper.

be seen in two different directions in terms of associations of individuals of type $m$ (males) and of type $f$ (females), as follows:

(i) From the classification matrix, we used a neural network approach which is different from the paper's SVM approach [13]:

(a) From the matrix, we can identify which colors have weak associations to emotions and which colors are typically confused with other colors.

(ii) Through an RGB regression, we study single emotion associations as well as how associations vary across age and gender ( $m$ and $f$ ), which we have not seen previously discussed through mathematical models. In particular, we see the following:

(a) Males typically associate the same emotions with darker colors than females do.

(b) Older people do associations to lighter colors.

We shall expand on the analysis and applications of the above findings in Sec. V.

\section{BACKGROUND: A MACHINE-LEARNING APPROACH TO QUANTIFY THE SPECIFICITY OF COLOR EMOTION}

The current research was inspired by the previous work in Ref. [13], and thus we shall dedicate this section to reviewing some of the main results which shall prove useful for our research and for comparison with our results. Their research proposes that the color specificity of emotion associations and country specificity of color-emotion associations can be measured using a multivariate pattern classification approach.

When classifying the data, the authors used an optimized support vector machine (SVM) approach with a 10-fold cross validation $(\mathrm{CV})$ to evaluate accuracy and considered two classifiers.

(i) The first classifier predicted color on the basis of 20 ratings of color-emotion associations. The classifier achieved an accuracy of $38.7 \%$ when tested on 4 countries, and achieved an accuracy of $30.4 \%$ when the classifier was applied to a dataset of 30 countries.

The true positive rate was the highest for black and red, followed by brown, pink, and gray. Thus they elicited very specific associations.

(ii) The second classifier predicted country on the basis of 240 ratings of color-emotion associations to quantify the degree to which color-emotion associations are country specific. The same learning model was used and achieved an accuracy of $80.2 \%$ when tested on the four countries China, Greece, Germany, and United Kingdom.

The data indicated strong country-specific color-emotion associations. For example, the association between brown and disgust was stronger in Germany than the other remaining countries, and almost nonexistent in China, and only the participants in Greece indicated a strong association between purple and sadness.

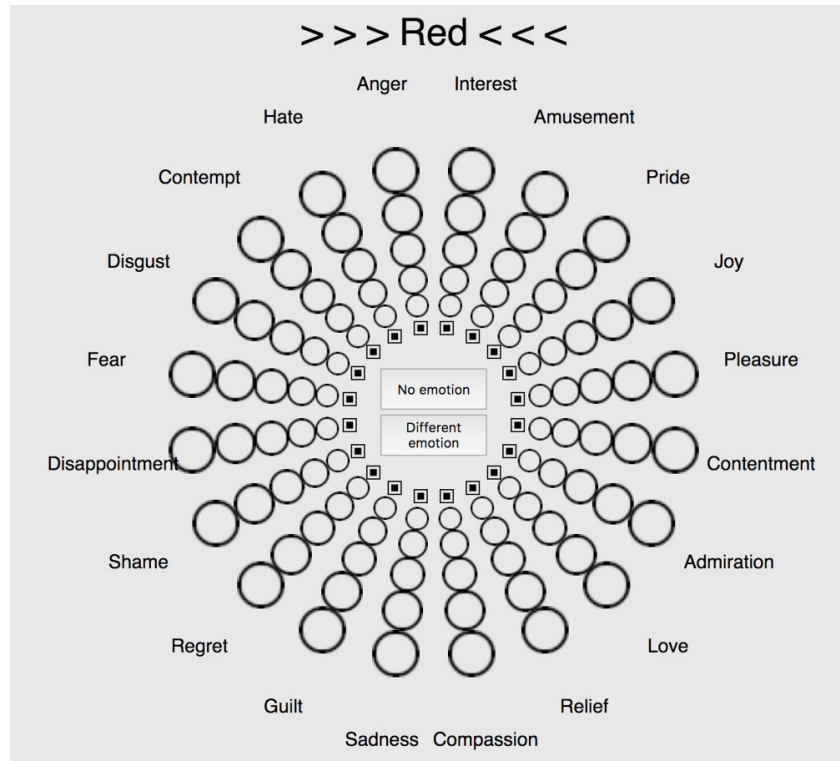

FIG. 3. Example of the platform within the survey used to collect the data in [12].

The data used in the project come from Forsbase's (2019) dataset [12] obtained through an international color-emotion survey program conducted by Jonauskaite and Mohr. Since 2015, researchers from the Institute of Psychology at the University of Lausanne have been collecting data from an international color-emotion survey. The data are collected online via the online platform [17], through which participants indicated the emotion that they associated to different color words through a graph of options, as shown in Fig. 3.

The survey asks for basic information including age, gender, country of origin, and language. Then the user is presented with 12 color terms and is asked to choose one, several, or none of the 20 emotions they associate with these color terms. Participants also rate the intensity of each associated emotion, quantified by an integer from 0 to 5 . In the present paper, we shall use the data for a sample of Greek speakers from Greece, Greek speakers from Cyprus, Turkish speakers from Cyprus, and Turkish speakers from Turkey, comprising 944 participants.

\section{PREDICTING THE ASSOCIATED COLORS}

As mentioned before, the data we shall use in the present manuscript consist of 944 submissions from [12]. We shall consider the gender variables as indicating participants to be type $m$ (male) and type $f$ (female), and shall consider the standard Decimal Code (R,G,B) conversion of the colors' words used in [12] to carry out our mathematical study. Including age and gender, a total of 22 input variables were used to predict the associated color. The learning structure that was used was a neural network with two hidden layers of size 10 , and an output layer with 12 nodes, one for each color as depicted in Fig. 4.

The predicted color is the output node with the greatest activation. The neural network was trained using a training set of size 2000 and testing using a cross-validation set 


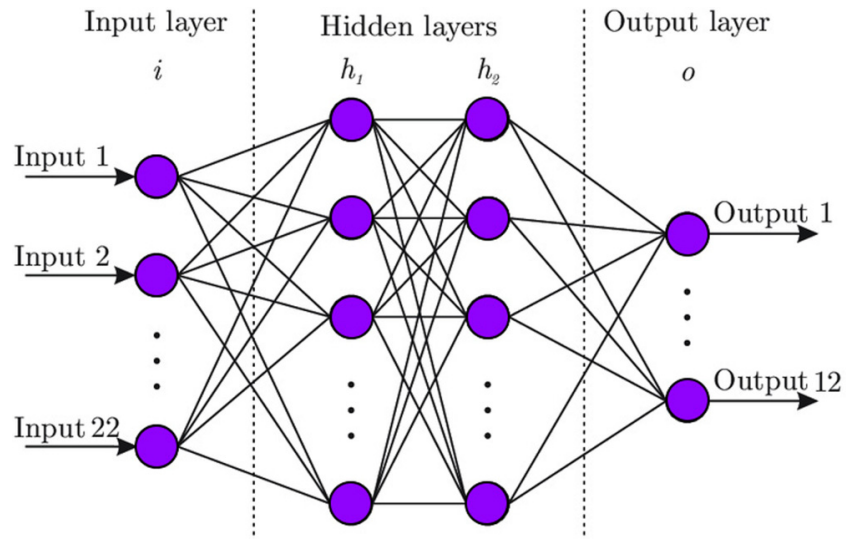

FIG. 4. Diagram of the neural network that was used.

of size approximately 10000 . We used the standard backpropagation algorithm to optimize the edge weight parameters of the neural network to improve the classification accuracy on the cross-validation set. The results are summarized in Fig. 5, with classification accuracy at about $33 \%$.

\section{A. Accuracy depending on colors}

From our study, we see different levels of accuracy obtained in our predictions, allowing us to infer the level of emotional association that colors have. Indeed, the following is observed, as depicted in Fig. 6:

(a) The colors black, brown, pink, and red are predicted with high accuracy and indicate a strong association with emotion.

(b) In contrast, the colors green and purple are predicted with very low accuracy and indicate weak association with emotion.

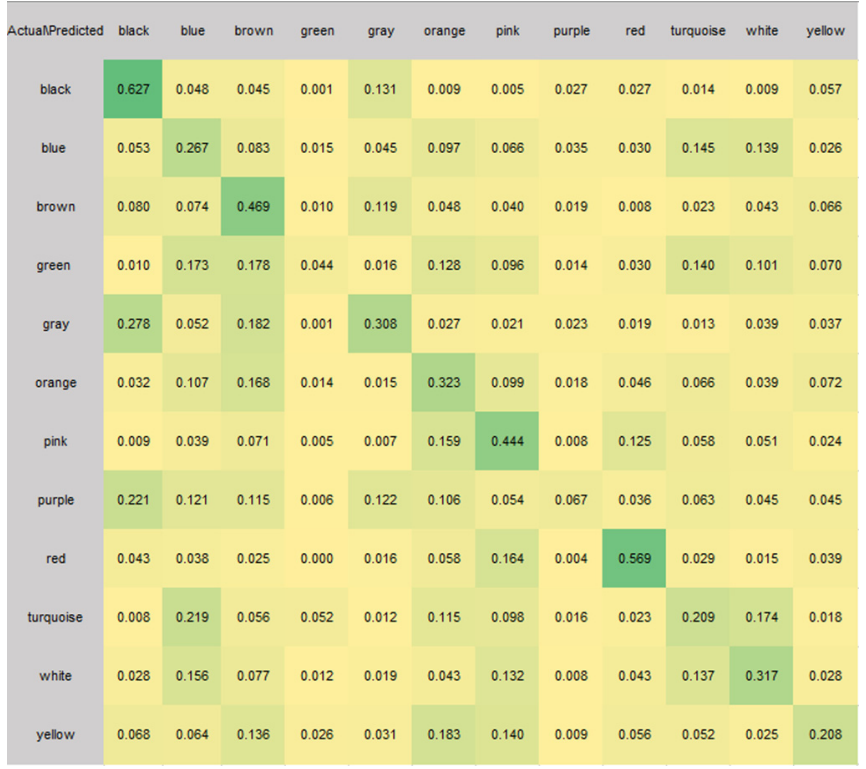

FIG. 5. Confusion matrix.

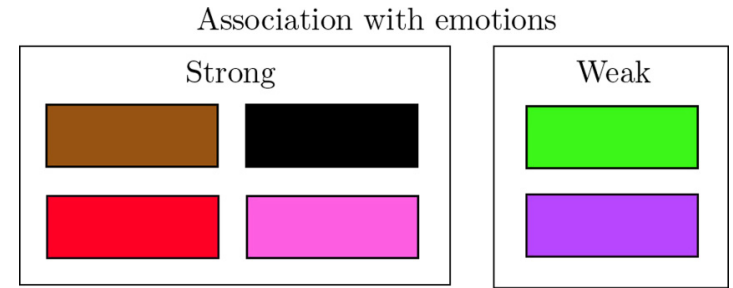

FIG. 6. Emotional association within discrete color analysis.

\section{B. Color exchanges}

Through our study, we note that some pairs of colors are frequently confused, indicating the variability of the emotional association and the need for further understanding the relation between colors in such pairs, both from the visual as well as emotional points of view. In particular, we note that as depicted in Fig. 7, the following occurs:

(a) black and brown are confused for gray;

(b) blue for turquoise and green;

(c) orange for yellow;

(d) brown for green and gray;

(e) and white for turquoise.

Interestingly, colors such as green and purple are rarely predicted at all, even though all colors appear the same number of times. This suggests that their association to emotion is weak enough to make it beneficial for the neural network to always output a different color. In order to improve accuracy, and given the above usual exchanges of colors, we consider the following two alternative setups to understand the color association:

(A) Group similar colors to minimize confusion and improve accuracy.

(B) Implement a continuous RGB output instead of a classification network. This can be used to demonstrate how subtle changes in color associations are influenced by varying emotion, and it should be noted that it was not attempted in [13].

\section{Grouping colors}

To improve the consistency of the color classification, one natural step is to reduce the dimension of the output layer by grouping colors by similar emotion associations in accordance with Fig. 8. In particular, we have grouped together the darker colors black, gray, and purple. By reducing the dimension by a factor of two, we have significantly improved the classification accuracy to $52 \%$ from the $33 \%$ in the full color classification. One notable observation is

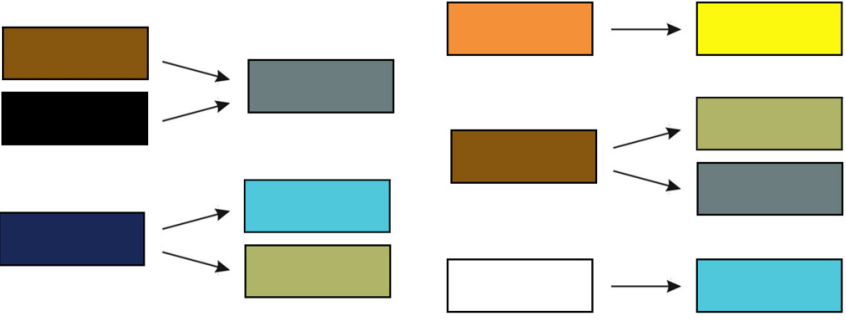

FIG. 7. Confusion exchanges. 


\begin{tabular}{|c|c|c|c|c|c|c|}
\hline $\begin{array}{c}\text { Actual\ } \\
\text { Predicted }\end{array}$ & $\begin{array}{c}\text { black } \\
\text { gray } \\
\text { purple }\end{array}$ & $\begin{array}{l}\text { blue } \\
\text { green } \\
\text { turquoise }\end{array}$ & brown & $\begin{array}{l}\text { orange } \\
\text { yellow }\end{array}$ & $\begin{array}{l}\text { pink } \\
\text { red }\end{array}$ & white \\
\hline $\begin{array}{c}\text { black } \\
\text { gray } \\
\text { purple }\end{array}$ & 0.632 & 0.209 & 0.023 & 0.102 & 0.032 & 0.003 \\
\hline $\begin{array}{c}\text { blue } \\
\text { green } \\
\text { turquoise }\end{array}$ & 0.084 & 0.699 & 0.029 & 0.116 & 0.053 & 0.018 \\
\hline brown & 0.310 & 0.320 & 0.243 & 0.111 & 0.010 & 0.005 \\
\hline $\begin{array}{l}\text { orange } \\
\text { yellow }\end{array}$ & 0.123 & 0.356 & 0.052 & 0.398 & 0.067 & 0.005 \\
\hline $\begin{array}{l}\text { pink } \\
\text { red }\end{array}$ & 0.065 & 0.184 & 0.014 & 0.168 & 0.550 & 0.019 \\
\hline white & 0.087 & 0.627 & 0.004 & 0.057 & 0.128 & 0.096 \\
\hline
\end{tabular}

FIG. 8. Confusion matrix for color groupings.

that the brown group is more often classified as the blackgray-purple group and the blue-green-turquoise group than its own group, a pattern not observed in the classification without dimension reduction. Additionally, the white group is almost never classified correctly, most often being classified as the blue-green-turquoise group. One possible explanation is that white is mostly associated with relief, a trait that is shared among the blue-green-turquoise group. Lastly, the orange-yellow group was incorrectly classified as the bluegreen-turquoise group about just as often as it was correctly classified, which again can be explained by shared emotions such as joy and pleasure.

\section{Average color association}

In the survey, participants are asked to describe the strength of the association between color-emotion pairs on a scale from 0 to 5. The table shown in Fig. 9 displays the average association value across every color-emotion pair among all the participants in the dataset, which serves as a visualization of the entire dataset without the age and gender factors. Some of the limitations of the results described in the single color association are that certain emotions can strongly associate with multiple conflicting colors, leading to an ambiguous mix of colors, often producing a brown. For example, both amusement and pleasure are associated with both colors such as blue and turquoise as well as colors such as red and orange, the combination of which cannot be described by a single color, leading to some ambiguity and variation.

Certain colors such as black, pink, and red display strong associations with at least one emotion, while others, such as brown and yellow, display weaker associations across all emotions. Likewise, we see that certain emotions such as love, relief, and sadness display strong associations with at least

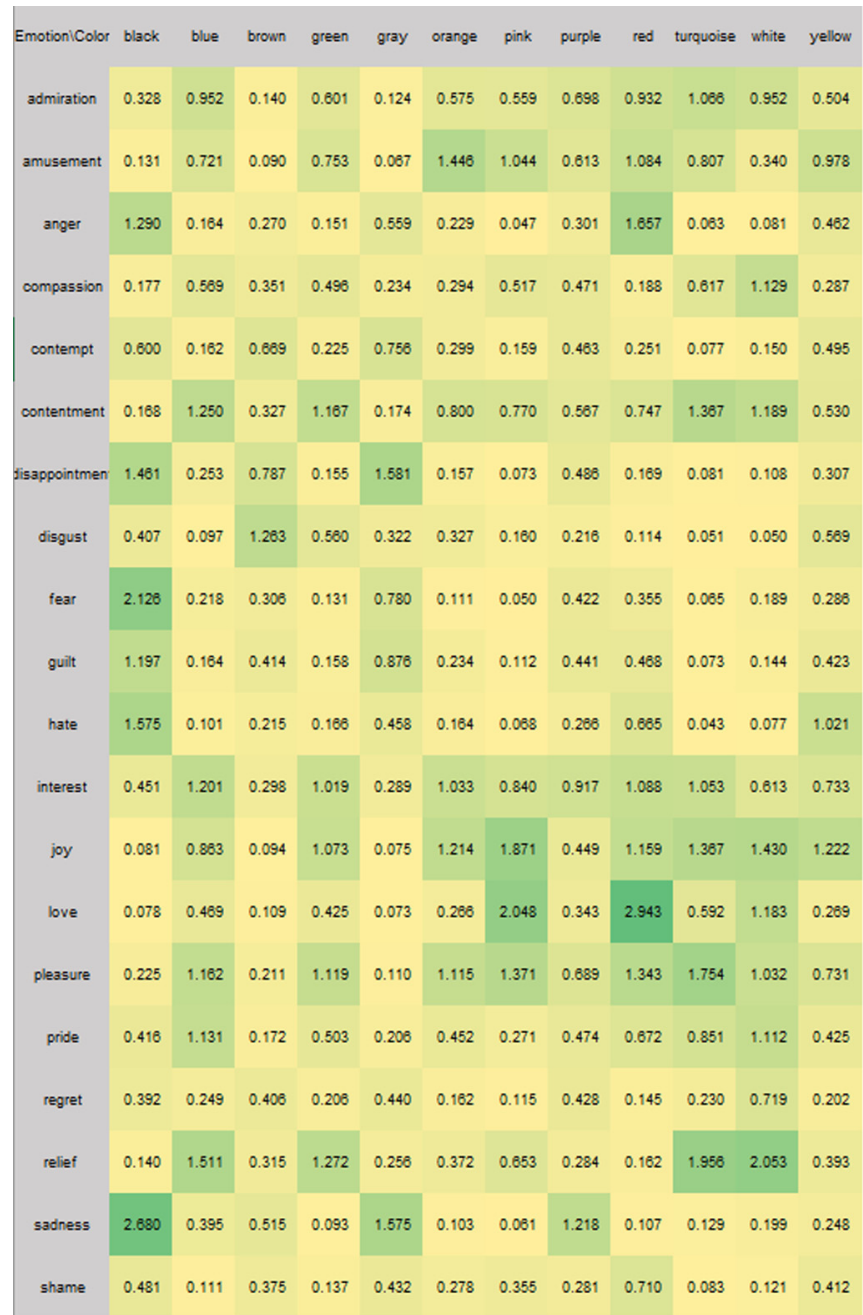

FIG. 9. Average color-emotion association.

one color, while others, such as contempt, regret, and shame, display weaker associations across all colors.

\section{CONTINUOUS RGB ANALYSIS}

The use of a continuum of colors for studying human emotional associations has appeared in the literature for many decades now (see, for example, the use within neural networks in [18], within emotional expressions in robotics [19], and when considering color combinations in [20]) and has become more important recently because of their impact in image-retrieval processes. While many experiments have used color-emotion metrics for single colors or pairs of colors, in many cases similar metrics were recently used in image retrieval showing that humans perceive color emotions for multicolored images in similar ways (e.g., see [21]). Hence, it becomes relevant to analyze the continuum of colors in the context of [12] to deduce novel correlations between colors and emotions.

The RGB regression study was implemented with the same neural network depicted in Fig. 4, with 22 nodes, two hidden layers of size 20 and 10, and three output nodes, representing the intensity of red, green, and blue. The training set (size 


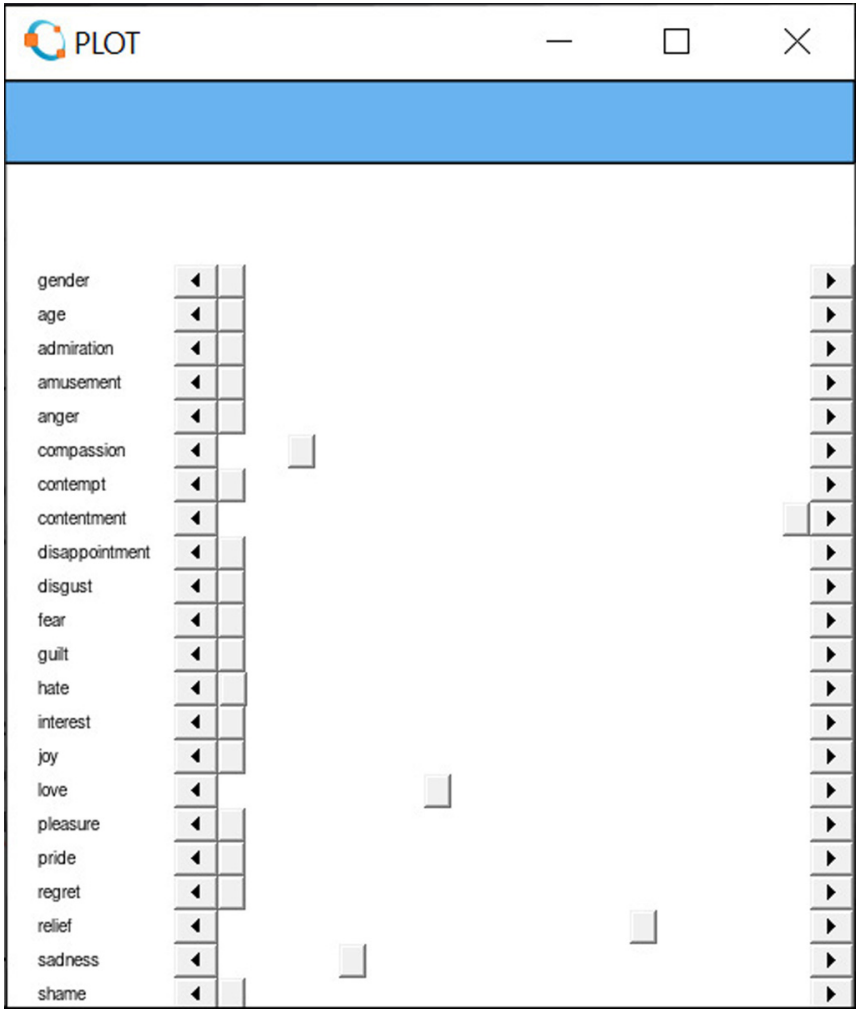

FIG. 10. RGB regression interface.

2000) was modified so that each color was converted to an RGB value based on standard conventions for color. By means of the neural network described above, we develop an interface which allows one to predict the color depending on the choice of different variables. This interface, shown in Fig. 10, has a slider for each input variable which can be adjusted. The age slider varies from 0 to 50, the gender slider varies from 0 to 1 ( 0 is female, 1 is male), and each emotion varies from 0 to 5 . The color at the top represents the predicted color based on the RGB output.

\section{A. Color-emotion associations across gender}

Gender differences when encoding and decoding color associations and facial emotions have long been considered (e.g., see [22] and references therein). While in many cases differences have been found, in other settings gender and age have not presented much difference. For example, gender and age were shown to be determining factors in the selection of achromatic black [23], while there were no gender differences in the main emotions linked to red among the children considered in [24].

By adjusting the gender input in the interface of Fig. 10, one can compare color-emotion associations across gender, as shown in Fig. 11. Indeed, through the regression, we find that females associate admiration with a dark purple, while males associate it with a dark blue. Across most emotions, we find the following:

(a) Both genders typically associate with similar emotions and colors.

\begin{tabular}{|c|c|c|}
\hline emotion & color (female) & color (male) \\
\hline admiration & & \\
\hline amusement & & \\
\hline anger & & \\
\hline compassion & & \\
\hline contempt & & \\
\hline contentment & & \\
\hline disappointment & & \\
\hline disgust & & \\
\hline fear & & \\
\hline guilt & & \\
\hline hate & & \\
\hline interest & & \\
\hline joy & & \\
\hline love & & \\
\hline pleasure & & \\
\hline pride & & \\
\hline regret & & \\
\hline relief & & \\
\hline sadness & & \\
\hline shame & & \\
\hline
\end{tabular}

FIG. 11. Gender (type $m$ and $f$ ) mathematical comparison in single color associations (for age $=20$ ).

(b) Exceptions include admiration, in which females associate with a light purple, while males associate with a blue;

(c) and regret, which females associate with a dark pink, while males associate with a red-orange.

Through our study, we also found that females typically associate emotions with lighter shades. This is most clearly seen in the following:

(a) anger, compassion, disgust, guilt, pleasure, pride, relief, and sadness;

(b) exceptions, in which cases males choose lighter colors, include shame and disappointment.

Interestingly, this agrees with experiments done for young ages, where young boys were more likely to associate positive emotions with darker colors than girls; e.g., see [24].

\section{B. Color-emotion associations across age}

By adjusting the age input, we can compare color-emotion associations across age, as shown in Fig. 12. Through our mathematical model, we find the following:

(a) Younger people associate anger with a darker shade of red, while older people associate it with a lighter shade.

(b) Older people associate most emotions with lighter shades than younger people. Examples are anger, compassion, hate, interest, and love.

(c) On the other hand, older people associate relief with a darker blue.

(d) There exist some emotions for which we see a consistent color across the age spectrum, such as contempt, guilt, joy, sadness, and shame. 


\begin{tabular}{|c|l|l|l|l|l|}
\hline emotion & 10 & 20 & 30 & 40 & 50 \\
\hline admiration & & & & & \\
\hline amusement & & & & & \\
\hline anger & & & & & \\
\hline compassion & & & & & \\
\hline contempt & & & & & \\
\hline contentment & & & & & \\
\hline disappointment & & & & & \\
\hline disgust & & & & & \\
\hline fear & & & & & \\
\hline guilt & & & & & \\
\hline hate & & & & & \\
\hline interest & & & & & \\
\hline joy & & & & & \\
\hline love & & & & & \\
\hline pleasure & & & & & \\
\hline pride & & & & & \\
\hline regret & & & & & \\
\hline relief & & & & & \\
\hline sadness & & & & \\
\hline shame & & & & & \\
\hline & & & \\
\hline
\end{tabular}

FIG. 12. Age comparison in single color associations (gender neutral), where shades of colors are obtained through the standard Decimal Code (R,G,B) obtained through our algorithm associated to each emotion.

Some emotion shifts are associated with a more brown color as age increases, as in admiration and contentment, which could indicate ambiguity, as in multiple color associations, or simply a lack of data as the age increases, leading to extrapolation. We shall return to this in Sec. V, where we compare our results to those from experimental papers which have looked into aging and color-emotion associations [25-27].

It is interesting to note that in the present manuscript, we have considered responses from Greek speakers from Greece, Greek speakers from Cyprus, Turkish speakers from Cyprus, and Turkish speakers from Turkey, and thus generically we get anger associated with shades of red. However, this should not hastily be generalized to a broader population: indeed, there have been studies such as [28] showing that people from Poland connected anger, envy, and jealousy with purple instead of red. In that paper, it is suggested that "cross-modal associations originate in universal human experiences and in culture-specific variables, such as language, mythology, and literature," and it would indeed be interesting to design a neural network to discern those differences once data from different regions is readily available.

\begin{tabular}{|c|c||c|c|}
\hline emotion & color & emotion & color \\
\hline \hline admiration & & hate & \\
\hline amusement & & interest & \\
\hline anger & & joy & \\
\hline compassion & & love & \\
\hline contempt & & pleasure & \\
\hline contentment & & pride & \\
\hline disappointment & & regret & \\
\hline disgust & & relief & \\
\hline fear & & sadness & \\
\hline guilt & & shame & \\
\hline
\end{tabular}

FIG. 13. Single emotion associations predicted through our model (age $=20$ ), where shades of colors are obtained through the standard Decimal Code (R,G,B) obtained through our algorithm associated to each emotion.

\section{Emotions associated to single colors}

Through our study, we can classify emotions into two distinguished sets: those that have a distinct association to a specific color and those that do not. Indeed, emotions such as anger, disgust, fear, love, pride, relief, and sadness have very clear and distinct associations to a specific color. However, other emotions such as amusement, compassion, joy, and shame may be associated with multiple colors and therefore provide an ambiguous color, generally a shade of brown or some arbitrary mix of colors. Figure 13 displays the color associated with a single emotion at maximum intensity among the gender neutral and average age setting (e.g., pure relief is most associated with a light-blue color, while pure anger is associated with a red color).

\section{CONCLUDING REMARKS}

In the present paper, we have analyzed a dataset provided by the online survey [12] in which 944 participants were presented with a series of 12 colors and asked to determine the association of each color with a set of 20 emotions in a scale from 0 to 5, via a platform depicted in Fig. 3. We used a machine-learning method to quantify the strength of color-emotion associations as well as their variation across age and gender. To quantify the strength of color-emotion associations, we employed a neural network to classify colors based on the participants' 20 emotion association values as well as their age and gender. The network consists of 22 input nodes and 12 output nodes (for each color), and two hidden layers of 10 nodes, as depicted in Fig. 4. The results of the classification based on a cross validation of 10000 , and on a training set of 2000, are summarized by the confusion matrix in Fig. 5.

In particular, we found that black, brown, pink, and red are classified correctly with high accuracy, indicating a strong association with specific emotions, while green and purple are rarely predicted and classified accurately, indicating a weak association with emotion (see Fig. 6). In order to improve accuracy, we combined similar colors and formed six color groups to reduce confusion among similar colors and ran 
an identical neural network to classify each group based on their corresponding emotion associations, increasing the classification accuracy to $52 \%$ (see Fig. 8). In particular, we find the following:

(I) brown is typically confused with the other darker colors, and

(II) both white and the orange-yellow group are notably confused with the blue-green-turquoise group.

We expect that this is likely due to shared associations with positive/negative emotions. We also investigated a neural-network-based regression to associate emotion and age/gender parameter colors on a continuous RGB spectrum. We used a neural network with the same 22 input nodes, but with three output nodes representing the red, green, and blue values. The network also consisted of two hidden layers of 20 and 10 nodes, and was trained on 2000 examples from the survey. We implemented an interface to display the output of the regression based on the 22 input variables, as shown in Fig. 10.

Using the regression, we determined precise colors in the Decimal Code $(\mathrm{R}, \mathrm{G}, \mathrm{B})$ associated with single emotions and its variation due to age and gender. The colors associated with single emotions (with age set to 20 and gender neutral) are summarized in Fig. 13. Most colors which were associated with multiple (conflicting) colors such as amusement and shame produced ambiguous colors, generally a shade of brown or an arbitrary mix of colors. In Fig. 11, we displayed the effects of gender on single emotion associations. Through the regression, we show that

(III) in most cases, females associated the same emotion with lighter colors, with exceptions including shame and disappointment.

In particular, Fig. 12 displaces the effects of age on single emotion associations. The different perception of colors and emotional associations has long been considered (e.g., see $[23,24,29])$. In particular, from experimental data, it is seen that bright colors have mainly positive emotional associations and dark colors have mainly negative emotional associations when not taking gender into account, but women responded more positively than men to bright colors and more negatively to dark colors [29]. From our mathematical study, as seen in item (III) above, we see that females indeed choose brighter colors than men for most positive emotions and choose darker colors than men for the most negative emotions, which is in agreement with the experimental results. However, there are still some negative emotions for which women tend to choose lighter colors than men, and thus it would be very interesting to pursue an experimental study of a detailed color association to a large range of negative emotions across gender.

When considering age differences, we found that older people associate most emotions with lighter shades of the same color, with relief being an exception. It is interesting to highlight the different effects of age on color association:

(IV) Some emotions such as contempt, guilt, joy, sadness, and shame were associated with similar shades across age.

(V) On the other hand, some emotions such as admiration and pleasure shift to a more brown color as age increases, indicating ambiguity or a lack of data (extrapolation).
In recent decades, many studies have found that older people are less able than young people to identify facial expressions $[25,26]$. Moreover, since it has been hypothesized that the emotional association of colors could be related to their association with facial expressions [27], one can expect the association of color to get tinted with age, leading to further ambiguity, as we have found in our study through both items (I) and (V) above. It would indeed be very interesting to carry out experimental research on how aging leads to a higher probability of brown being associated with emotions.

Finally, considering the findings in terms of cross-age and gender color association, one may consider their implications within marketing strategies of different kinds. An example of this is, for instance, when marketing perfumes for different ages and genders. In such cases, one can use the colors people tend to associate with their smells for the packaging [30], and we propose that further considering their association with emotions could lead to more targeted audiences.

When considering single color association, certain emotions strongly associate with multiple conflicting colors, leading to an ambiguous mix of colors (often a brown) output by the regression. This ambiguity can also explain some of the variation found in the age and gender spectrum analysis. For example, both shame and admiration have relatively weak associations across all colors, which could explain some of the variation shown in the gender comparison. It should also be noted that in the RGB regression, different instances of the training lead to slightly different results in the colors produced. To reduce variation, we train using a high number of iterations (1000) and increase the standard regularization parameter $\lambda$ (incorporated to avoid the risk of overfitting) to achieve the most consistent results.

\section{ACKNOWLEDGMENTS}

The leading authors V.R. and L.P.S. are thankful to MIT PRIMES-USA for the opportunity to conduct this research together, and in particular Tanya Khovanova for her continued support and James Unwin for insightful comments on a draft of the manuscript. Moreover, they greatly acknowledge the work of their co-authors to produce Ref. [12]. The work of L.S. is partially supported through the NSF Grant No. CAREER DMS 1749013. The Swiss National Science Foundation supported the work of D.J. with the Doc.CH fellowship Grant No. P0LAP1_175055 and C.M. with a project Grant No. 100014_182138.

V.R. and L.P.S. carried out the research and preparation of the present manuscript. The remaining authors were responsible for the data source. In particular, N.K. was responsible for the Greek Cypriot data of [12], E.V. was responsible for the Turkish Cypriot data of [12], M.P.-P. was responsible for the Greek data of [12], B.M. was responsible for the Turkish data of [12], and C.M. and D.J. were responsible for the overall data conceptualization of [12], coordination of the translations, data acquisition, and research dissemination. 
[1] M. Luckiesh, The Language of Color (Dodd, Mead, 1918).

[2] S. Mastandrea, How Emotions Shape Aesthetic Experiences, Cambridge Handbooks in Psychology (Cambridge University Press, Cambridge, 2014), pp. 500-518.

[3] G. H. Taylor, J. Abnorm. Soc. Psychol. 7, 347 (1912).

[4] L. B. Wexner, J. Appl. Psychol. 38, 432 (1954).

[5] F. M. Adams and C. E. Osgood, J. Cross-Cultural Psychol. 4, 135 (1973)

[6] K. Naz and H. Epps, Coll. Stud. J. 38, 396 (2004).

[7] S. E. Palmer and K. B. Schloss, Proc. Natl. Acad. Sci. 107, 8877 (2010).

[8] K. R. Scherer, V. Shuman, J. Fontaine, and C. Soriano Salinas, The GRID meets the wheel: assessing emotional feeling via self-report (2013), https://www.semanticscholar. org/paper/The-GRID-meets-the-wheel\%3A-assessingemotional-via-Scherer-Shuman/032d8f32c1b7d40ee1298 1ebda51229578e75f32.

[9] See, for instance, John James Audubon ("The Birds of America") journal from 1827 where he wrote that he "had the blues."

[10] Origins of Navy Terminology, https://www.navy.mil/navydata/ traditions/html/navyterm.html (unpublished).

[11] D. Jonauskaite, A. M. Abdel-Khalek, A. Abu-Akel, A. S. AlRasheed, J.-P. Antonietti, Á. G. Ásgeirsson, K. A. Atitsogbe, M. Barma, D. Barratt, V. Bogushevskaya et al., J. Environ. Psychol. 66, 101350 (2019).

[12] D. Jonauskaite, C. Mohr (PI), and N. Dael, International colouremotion survey (2020), https://forsbase.unil.ch/project/studypublic-overview/15126/0/.

[13] D. Jonauskaite, J. Wicker, C. Mohr, N. Dael, J. Havelka, M. Papadatou-Pastou, M. Zhang, and D. Oberfeld. R. Soc. 6, 190741 (2019).

[14] P. Valdez and A. Mehrabian, J. Expt. Psychol.: Gen. 123, 394 (1994).

[15] L. Wilms and D. Oberfeld, Psychol. Res. 82, 896 (2018).
[16] Where RGB stands for when red, green, and blue lights are added together in various ways to reproduce a broad array of colors.

[17] https://www2.unil.ch/onlinepsylab/colour/main.php.

[18] M.-F. Lee and G.-S. Chen, Backpropagation neural network model for detecting artificial emotions with color, International Joint Conference on Awareness Science and Technology \& Ubi-Media Computing (iCAST 2013 \& UMEDIA 2013) (IEEE, 2013), pp. 433-438.

[19] M.-g. Kim, H. S. Lee, J. W. Park, S. H. Jo, and M. J. Chung, Determining color and blinking to support facial expression of a robot for conveying emotional intensity, RO-MAN 2008-The 17th IEEE International Symposium on Robot and Human Interactive Communication (IEEE, 2008), pp. 219-224.

[20] G. Csurka, S. Skaff, L. Marchesotti, and C. Saunders, Learning moods and emotions from color combinations, Proceedings of the Seventh Indian Conference on Computer Vision, Graphics and Image Processing (ACM, 2010), pp. 398-305.

[21] M. Solli and R. Lenz, Color Res. Appl. 36, 210 (2011).

[22] N. G. Rotter and G. S. Rotter, J. Nonverbal Behav. 12, 139 (1988).

[23] B. Manav, Color Res. Appl. 32, 144 (2007).

[24] C. J. Boyatzis and R. Varghese, J. Genet. Psychol. 155, 77 (1994).

[25] L. H. Phillips, R. D. MacLean, and R. Allen, J. Gerontol. Ser. B: Psychol. Sci. Soc. Sci. 57, P526 (2002).

[26] N. C. Ebner and M. K. Johnson, Emotion 9, 329 (2009).

[27] M. R. Zentner, Dev. Sci. 4, 389 (2001).

[28] R. B. Hupka, Z. Zaleski, J. Otto, L. Reidl, and N. V. Tarabrina, J. Cross-Cultural Psychol. 28, 156 (1997).

[29] M. Hemphill, J. Genet. Psychol. 157, 275 (1996).

[30] H. N. Schifferstein and I. Tanudjaja, Perception 33, 1249 (2004). 